\title{
¿Descubrir o intervenir? E1 conocimiento de la interculturalidad en la educación superior de Brasil y México: politicas y sujetos interculturales como objetos de reflexión y conocimiento ${ }^{1}$
}

\section{Mario A. Martínez Cortés ${ }^{2}$}

Lateinamerika Institut de la Universidad Libre de Berlín, Berlín, Alemania $^{3}$

mariomtz@zedat.fu-berlin.de

Recibido: 7 de febrero de 2014

Aceptado: 3 de junio de 2014

Disponible en línea: 15 de marzo de 2015

\footnotetext{
1 Artículo de investigación en torno a los resultados de mi investigación doctoral en Sociología. En él se hace una reflexión general sobre los resultados obtenidos sugiriendo algunas interpretaciones, de modo que no se ahonda en los detalles que componen operativamente las categorias de análisis empleadas. Esta investigación fue posible gracias al financiamiento de la Dahlem Research School de la Universidad Libre de Berlín y del programa de Becas CONACYT al Extranjero - 2012 (México).

2 Licenciado en Psicología, UNAM (México), 2002. Maestro en Estudios Sociales sobre Educación Superior, Universidad de Kassel (Alemania), 2008

3 Estudiante de Doctorado en Sociología
} 


\title{
¿Descubrir o intervenir? E1 conocimiento de la interculturalidad en la educación superior de Brasil y México: politicas y sujetos interculturales como objetos de reflexión y conocimiento
}

\begin{abstract}
Resumen
Se plantea una aproximación proximal-performativa a las politicas de interculturalidad en educación superior de dos casos, Brasil y México. Se sugiere que un elemento de diferenciación de estas es el conocimiento que producen. De manera específica, se considera que, aunque el conocimiento de la interculturalidad contiene elementos comunes en los dos casos analizados (lo intercultural como algo distinto a los conocimientos indígenas, la abierta defensa de intereses indigenas y la noción de participación) que corresponden a una vertiente estática, existen elementos que permiten su diferenciación (la problematización de la opción intercultural como tema de reflexión y el origen actoral de las propuestas), a través de los cuales estas políticas se pueden convertir en proyectos de llegar-a-ser, brindando esperanzas y posiciones estratégicas más que soluciones acabadas. Estas reflexiones parten del análisis de documentos de divulgación del conocimiento producido dentro de políticas específicas: programa PROLIND de Brasil y universidades interculturales de México.

Palabras clave: interculturalidad; etnicidad; reconocimiento; educación superior; conocimiento; Pueblos Indigenas

\section{Discover or Intervene? Interculturality Knowledge in Brazil and Mexico higher Education: Policies and Intercultural Subjects as Objects of Reflection and Knowledge}

\begin{abstract}
A proximal-performative approach to the intercultural policies in two cases of higher education in Brazil and Mexico is layed out. It is suggested that a differentiating element between these is the knowledge they produce. Specifically, it is considered that, although intercultural knowledge contains common elements in the two cases analyzed (intercultural as something different from the indigenous knowledge, the open defense of indigenous interests and the notion of participation) that correspond to a static vision, there are elements that allow their differentiation (the problematization the intercultural option as a theme for reflection and the acting origin of proposals), through which these policies can be converted into projects, providing hope and strategic positions instead of closed solutions. These reflections are based on the analysis of documents for the dissemination of knowledge produced within specific policies: PROLIND program from Brazil and intercultural universities in Mexico.
\end{abstract}

Keywords: interculturality; ethnicity; recognition; higher education; knowledge; Indigenous people

\section{Descobrir ou Intervir? O conhecimento da interculturalidade no ensino superior do Brasil e o México: políticas e sujeitos interculturais como objetos de reflexão e conhecimento}

\begin{abstract}
Resumo
Levanta-se abordagem proximal-performativa às politicas de interculturalidade em ensino superior de dois casos, o Brasil e o México. Sugere-se que um elemento de diferenciação daquelas é o conhecimento que produzem. De maneira específica, considera-se que, embora o conhecimento da interculturalidade contém elementos comuns nos dois casos analisados (o intercultural como algo diferente aos conhecimentos indígenas, a defensa aberta de interesses indígenas e a noção de participação) que correspondem a una vertente estática, existem elementos que permitem sua diferenciação (a problematização da opção intercultural como tema de reflexão e a origem atoral das propostas), através dos quais estas politicas podem virar para projetos de chegar-a-ser, dando esperanças e posições estratégicas mais que soluções acabadas. Estas reflexões partem da análise de documentos de difusão do conhecimento produzido dentro de políticas específicas: programa PROLIND do Brasil e universidades interculturais do México. Palavras-chave: interculturalidade; etnicidade; reconhecimento; ensino superior; conhecimento; Povos Indigenas
\end{abstract}




\section{Interculturalidad y conocimiento: ¿democratización o distracción?}

Cuando se habla de interculturalidad en educación superior en América Latina se hace referencia a soluciones sociopoliticas orientadas a reducir la exclusión de poblaciones indígenas en ese nivel educativo, no solo a través de una inclusión numérica de individuos, sino también en términos cualitativos por medio de la valoración positiva de aquello que durante la construcción de las naciones modernas trató de negarse: la diferencia cultural indígena.

En ese sentido, la noción de interculturalidad conecta con la de conocimiento de dos formas: como medio para redistribuir el acceso al conocimiento y al entrenamiento en educación superior y como medio para reconocer el valor simbólico de las culturas indígenas y sus conocimientos. Así, dentro de la ola de democratización en América Latina protagonizada por las poblaciones indígenas (Domingues, 2009; Trejo, 2006/2000), la interculturalidad ha llegado a ser un término primordial. Las propuestas interculturales revelan una transformación significativa en la historia de los Estados latinoamericanos y su visión sobre la justicia con respecto a grupos culturalmente diferenciados que supera la idea de una ciudadanía universal donde la identificación étnica pertenecía al terreno de lo privado. Dicha transformación se refleja en la transición gradual de los sistemas educativos que a inicios del siglo XX no contaban con medidas específicas para la diferencia cultural, pero que para fines del mismo habian incorporado dicha noción como aspecto valioso per se y no solo como recurso pedagógico (López, 1994; 2001; Ströbele-Gregor, 2010).

Sin embargo, hay todavia una tercera forma en la que podemos relacionar al conocimiento con la interculturalidad y es esta desde donde la presente investigación se desarrolla. A diferencia de las dos primeras, esta tercera forma de relación no se refiere al conocimiento como algo exterior, más bien se trata de una mirada deconstructiva a las nociones de conocimiento que la idea misma de interculturalidad produce. El concepto de reconocimiento ayuda a aclarar las implicaciones de esta tercera relación. 
En las dos primeras aproximaciones, el conocimiento prácticamente existe como objeto acabado, de modo que puede ser redistribuido o reconocido. Bajo esa perspectiva de existencia, la crítica a las políticas gubernamentales de educación intercultural señala con pericia que el reconocimiento de otros conocimientos por las instituciones de la sociedad mayoritaria no-indígena resulta equivocado. Para los defensores de la igualdad, las políticas de interculturalidad, al concentrarse en conocimientos no occidentales, no redistribuyen los bienes adecuados para reparar las injusticias que los Pueblos Indigenas padecen (por ejemplo, el conocimiento indígena parece poco prometedor en el alivio de la pobreza).

Por otro lado, los defensores del reconocimiento de la diferencia argumentan que la institucionalización de los conocimientos indígenas en sistemas formales de educación contribuye a la apropiación y mal uso de los primeros por parte de actores que no representan a los intereses indigenas. De acuerdo con estas críticas, el reconocimiento que promueve la interculturalidad centrándose en el conocimiento indígena estaría equivocado porque desvía a las poblaciones de los conocimientos verdaderamente necesarios para un cambio social o porque actúa en contra de lo que parece ser la esencia de los conocimientos indígenas al descontextualizarlos y comercializarlos como productos educativos.

Aunque válidas, estas críticas solo dejan ver que la interculturalidad como política de reconocimiento falla, siendo ante todo una nueva estrategia de dominación. Esto constituye lo que Latour (2004) llama una posición romántica (fairy), donde la crítica se limita a mostrar que las personas juegan el rol de creyentes naïve que proyectan sus deseos sobre distintos objetos del mundo convirtiéndolos en fetiches. Tal posición elimina toda complejidad, pues no nos deja ver el lado estratégico de la aceptación de las políticas, que como menciona Walsh (2002), implica una lógica simultánea de estar fuera-dentro-en contra.

Estas críticas muestran además, una debilidad que tiene que ver con la noción de conocimiento, lo que no se puede pasar por alto precisamente porque ello implicaría dejar intactas ciertas premisas a través de las que la injusticia contra los Pueblos Indígenas ha operado siste- 
máticamente desde el siglo XVI y que han sido denunciadas por intelectuales y movimientos sociales. Puntualmente, una visión que define al conocimiento como un objeto, una serie de contenidos, es también una visión que está sustentada, y a la vez sustenta, al capitalismo global. Al respecto, Quijano (2007) nos indica que para el capitalismo, el conocimiento se define en términos de propiedad privada, como una relación directa entre un sujeto humano y los objetos que forman parte del mundo, cuando se trata más bien de una relación entre sujetos acerca de esos objetos.

Esta aproximación es interesante porque permite descentrar la defensa de la diferencia cultural con respecto al conocimiento de las meras características identitarias y pone atención a las asimetrias en las relaciones de poder. La inclusión de estas en el análisis permite revelar que la definición de qué es conocimiento es histórica y por tanto, que a través de la creación de límites raciales, civilizatorios y étnicos -aunados a los de género- ciertos grupos han sido autorizados y naturalizados como productores de conocimiento mientras otros no.

Cuestionar la noción misma de diferencia resulta útil para ese descentramiento. Desde diversas perspectivas y disciplinas hoy sabemos que la diferencia es una construcción histórica donde los límites entre un 'nosotros' y uno o varios 'otros' son establecidos. De ese modo, las identidades son productos de procesos sociales y es en ese sentido en que la justicia basada en el reconocimiento debiera tomar relevancia avanzando a un nivel menos superficial que la identidad acabada que se transmite de generación en generación, lo cual siempre es una simplificación (Abu-Lughod, 1997; Grimson y Semán, 2005; Ortner, 2006; Restrepo, 2004a; 2004b). Así, se propone que hay que mirar lo que los defensores de esas identidades hacen para lograr el reconocimiento y también lo que hacen una vez que lo logran.

De ese modo, así como con el 'conocimiento', la noción de reconocimiento debiera verse menos como una relación entre un sujeto y el mundo exterior -que por ser exterior es objetivo e independiente- y apelar más a las relaciones entre personas donde lo que se reconoce nunca es algo cerrado sino que está en constante negociación y evolución. Solo 
de ese modo se reconoce la agencia del sujeto, su capacidad de hacer. En ese sentido, reconocimiento no solo envuelve la noción de acknowledgement o la aceptación de la existencia de algo diferente y una subsecuente valoración positiva, sino también la capacidad de interpelación de esa posición subalterna que puede transformar la realidad del mundo que se comparte.

Volviendo así a la propuesta de Quijano, las identidades indígenas, como identidades raciales producto de la invasión Europea a lo que hoy es América, sirven como categoría de clasificación social para la distribución desigual del trabajo y sus productos así como de la autoridad y el estatus social. De ese modo, ya que la clasificación de la diferencia en diferentes grupos étnicos involucra desigualdad, también envuelve formas de jerarquización de la población. No solo existe una colonialidad del poder que articula raza y capitalismo sino también una colonialidad del saber (Lander, 2000) donde las poblaciones de la parte del mundo considerada periférica son vistas como meros receptores de los conocimientos verdaderos que explicarían el mundo y ayudarían así a producir desarrollo.

La descalificación epistémica (Maldonado-Torres, 2007) no es solo subalternización de otras prácticas culturales para conocer el mundo sino también la implementación de un sistema-mundo donde esos otros ocupan una permanente posición de desventaja, puesto que al mismo tiempo que se les niega y rechaza como diferentes, se les impide mejorar su posición dentro de la jerarquía capitalista al conducir una sistemática distribución desigual de bienes de conocimiento. En ese sistema cualquier opción ha resultado pérdida para los Pueblos Indígenas: si preservan su diferencia se les cierran posibilidades dentro de los parámetros socioeconómicos dominantes, si experimentan movilidad social y se hacen ciudadanos 'universales' confirman el discurso denigrante sobre lo indígena. Así, el reto de la noción de reconocimiento es desnaturalizar ese sistema donde la diferencia existe como tal, independiente de los otros y de las relaciones de poder que se tejen con ellos.

Este artículo argumenta que si miramos los alcances de la educación intercultural desde esta tercera forma de relación, podemos 
ver cómo las políticas de reconocimiento implican esfuerzos por desnaturalizar un sistema jerárquico de posiciones en la producción de conocimiento. Para las dos primeras formas externas de relación, las soluciones habian sido la redistribución del acceso al conocimiento hegemónico y el reforzamiento del vínculo entre tradiciones y prácticas de conocimiento específicas con grupos particulares de personas. En ambas soluciones la noción de conocimiento es problemática porque borra las relaciones de poder. En el primer caso, el conocimiento es una cosa específica capaz de poseerse, acumularse y transmitirse; en el segundo caso, la valoración del conocimiento diferenciado convierte al grupo cultural en el objeto reconocido en tanto congela la diferencia y homogeneiza a diferentes individuos bajo la idea de cultura.

Así, nos encontramos con dos grandes tendencias para pensar el conocimiento. Una representacional, donde estas dos primeras formas de relación interculturalidad-conocimiento hacen sentido y otra, que aquí se define como distal-performativa, donde el conocimiento que se produce en el marco de las politicas de reconocimiento deja de centrarse en descripciones objetivistas para enfocarse en la conducción de proyectos de transformación social que respondan a los intereses de las propias poblaciones target.

El conocimiento como representación se basa en la bien conocida división cartesiana entre objeto y sujeto de conocimiento, la cual caracteriza al pensamiento de la modernidad (Apffel-Marglin, 1996; CastroGómez, 2007; Castro-Gómez y Grosfoguel, 2007; Maldonado-Torres, 2007) y que calificó a las poblaciones indígenas como salvajes, carentes de civilización, subdesarrolladas y vestigios del pasado (Escobar, 1999; Fabian, 1983) contraponiéndolas a la idea de modernidad.

En esa versión clásica del conocimiento, las características del mundo existen como tales independientemente del observador y de acuerdo a ello, la forma a través de la que se da cuenta del mundo tendria que caracterizarse por ser objetiva, es decir, sin que los intereses de los sujetos de conocimiento puedan hacerse visibles en la definición de lo que se estudia, la selección de los métodos y la construcción de las conclusiones. 
Por otro lado, tenemos una perspectiva de conocimiento proximal-performativa que se diferencia claramente de la representacional. El término proximal es retomado de Cooper y Law (1995) quienes describen al pensamiento proximal como aquel que presta atención a la vida social como proceso. En lugar de dar por sentado lo constituido, esta perspectiva concibe a la realidad como algo que ocurre en forma continua, por tanto inacabada, aquello que es siempre aproximado pero nunca totalmente realizado. A diferencia del pensamiento distal -que sería el de la representación-, el pensamiento proximal no busca conocer las reglas universales del mundo para después hacer uso de las ciencias sociales como herramienta de la racionalidad moderna, es decir, para a través de ellas planear y organizar objetivamente el cambio social (Wallerstein, 1996); más bien reconoce que el conocimiento está mediado por la multiplicidad, la incertidumbre y los intereses (Wallerstein, 2004).

De esta manera, una perspectiva proximal del conocimiento descansa en la premisa de que más que conocimientos universales, siempre conocemos desde nuestra experiencia situada, la cual habremos de reconocer como parcial, en lugar de completa, pero no por ello como irreal. Así, la perspectiva proximal apela a la generación de puntos de vista o posiciones parciales en lugar de la idea estática de identidad (Haraway 1988; 1995; 2008; Strathern, 1991). Con respecto a la interculturalidad como solución social, se puede proponer que su potencial transformador radica entonces, más que en su objetividad y certidumbre, en las posibilidades de cambio que puede abrir en cierto momento. En ese sentido, la política correspondiente sería una de llegar-a-ser (becoming) más que una política de identidad.

El conocimiento que se produce en el marco de la interculturalidad, al ser un conocimiento marcado por intereses particulares que incluyen diferentes versiones de democratización, solo puede constituirse como parcialidad. Este conocimiento requiere además la noción de participantes parciales (Strathern, 1991): actores que se conectan solo en ciertos aspectos con otros, como podría ser con la opción intercultural misma, para así poder presentar demandas y ser escuchados (Haraway, 1995). 
Es por ello que, el potencial transformador del conocimiento se aborda aquí no como un poder de representación adecuado o preciso, cuyo fin es mapear y reflejar el mundo tal y como es (Pickering, 1995); sino como una actividad social que produce espacios donde se posibilita la producción de conocimiento (Turnbull, 1997), en este caso, por parte de sujetos históricamente descalificados para ello. Es en este sentido que se afirma que el conocimiento de la interculturalidad (cdi) involucra una posibilidad de transformación a partir del conocimiento, ya que al enfatizar la acción de los indígenas en su faceta de productores de conocimiento abiertamente politizado, la interculturalidad puede pasar de pensarse como solución social técnico-científica basada en la descripción objetiva de la diversidad cultural a la idea de solución social como colaboradora en y generadora de procesos sociopolíticos de mayor amplitud.

Además de su carácter inclusivo de intereses, se tiene que reconocer que parte importante del conocimiento que se produce por las políticas de interculturalidad en educación superior (PIES) gira en torno a los alcances que la misma educación intercultural ofrece. En ese sentido, esta constituye su cuerpo de conocimientos conforme es implementada; de ahí que se considere que además de proximal, una perspectiva transformadora de conocimiento intercultural es también performativa: no se limita a describir el mundo, sino a construir el objeto del cual produce conocimiento.

\section{El conocimiento de la interculturalidad}

En esta investigación se argumenta que las PIES envuelven una concepción de conocimiento distinta, de ahí que no se trate de políticas de redistribución, pero tampoco de una distinción de los conocimientos como rasgos culturales que definen a un grupo como étnico o tradicional. Más bien, se propone que la interculturalidad trata de conocimientos que se centran en el reconocimiento de sujetos indigenas como productores de conocimiento quienes, problematizando acerca 
de la diferencia cultural, revelan la búsqueda de un cambio de posición de la etnicidad indígena dentro de contextos nacionales.

Ese conocimiento llamado aquí conocimiento de la interculturalidad (cdi), no corresponde por tanto a una representación del mundo tal y como es, sino a la materialización de demandas politicas a través de proyectos de futuro que surgen de la implementación de las PIES, pero que al mismo tiempo, no se reducen a manifiestos políticos.

Ahora bien, aquí es preciso hacer una aclaración. Hablar del cdi como un 'tipo de conocimiento' distinto tanto a la representación del mundo como a las demandas políticas e intereses de grupos implica también una forma de reificación y por tanto, de reducción. Esta conceptualización sin embargo, es usada en esta investigación como un punto de entrada analítico, que sería de orden estático. No obstante, también se considera que el cdi puede envolver una vertiente dinámica que es donde radica su potencial transformador. A su vez, lo estático puede ser considerado como el discurso global sobre interculturalidad, mientras que lo dinámico se centra en la particularidad histórica local que dota de sentido a las ideas y prácticas de educación intercultural.

Desde una vertiente estática, el cdi nos señala que en el discurso intercultural las perspectivas del sujeto target de la politica pública y por ende de las demandas de justicia, son consideradas elementos obligatorios -al menos idealmente- para que las políticas que giran en torno al conocimiento puedan significar reconocimiento. Este razonamiento es una condición básica de las políticas de reconocimiento en educación que puede observarse aún en distintas latitudes, a través del énfasis que se pone en la promoción de modelos educativos participativos. La lógica es: si el sujeto adquiere un rol activo dentro de sus procesos educativos, entonces sus intereses serán satisfechos por la educación como solución social.

Sin embargo, esta formalidad discursiva en la que pueden coincidir diferentes proyectos, sean de estado o de movimientos sociales, no nos ofrece la posibilidad de adentrarnos en la interculturalidad de 
manera más dinámica. Es decir, como una solución que puede generar procesos y no estados, sujetos dinámicos y no estáticos. Esto es, el discurso de la participación deja de poner atención al sujeto una vez que sus demandas de reconocimiento son alcanzadas y materializadas en eventos específicos; de ese modo, la noción de reconocimiento alcanza un cierre convirtiéndose en un estado, donde nuevamente las relaciones asimétricas de poder parecen desaparecer. En resumen, la participación parece ser a primera vista la solución genérica que no presta atención a las particularidades con que el término es implementado en diferentes contextos; de ahí que visualice a la solución intercultural de forma estática, como algo que una vez constituido desde la ciencia social ofrecerá las soluciones deseadas.

La perspectiva dinámica argumenta que por medio de la noción del cdi, la interculturalidad puede ofrecer una alternativa a la naturalización del sistema jerárquico colonial en el área del conocimiento. Esto no solo porque presta atención a lo que los sujetos hacen ya en su posición de sujetos reconocidos (por ejemplo como estudiantes interculturales), sino porque al hacer de sí misma -y de sus alcances como solución social- un objeto de conocimiento y reflexión, la educación intercultural puede brindar una visión de conocimiento sobre la transformación social que puede resultar útil para los Pueblos indígenas, al basarse más en procesos dinámicos de llegar-a-ser que en estados fijos de identidades. Así, la identificación de una vertiente dinámica del cdi sirve para caracterizar diversos casos en los que la interculturalidad ha sido implementada como politica educativa formal mientras que la vertiente estática hace referencia a las coincidencias del discurso de interculturalidad en educación. A continuación se define más puntualmente, desde estas dos vertientes, lo que se entiende por cdi:

En el nivel de una vertiente estática, el cdi:

- no se refiere propiamente a los conocimientos o prácticas indígenas, sino que está ligado a las ideas de su rescate y preservación. Así, se trata de la materialización de indicaciones y premisas para poner en relación de diálogo a tales conocimientos con aquellos de la ciencia occidental; 
- proyecta claramente trabajar a favor de los intereses indígenas en sociedades que los han oprimido;

- se caracteriza como un conocimiento que enfatiza la acción y participación de los sujetos para que construyan proyectos de intervención a partir del conocimiento de su comunidad y de ese modo mejorar sus condiciones materiales, políticas y de respeto social.

En el nivel de una vertiente dinámica, el cdi:

- al ocuparse de la opción intercultural como objeto de conocimiento, es reflexivo. Con ello no solo apela al reconocimiento que los otros otorgan a los Pueblos Indígenas, sino que evidencia que el sujeto ya está actuando, demostrando sus capacidades y diferencias;

- da relevancia al origen de las iniciativas de participación de modo que no da por sentado que el decir que se habla desde los intereses indigenas implique necesariamente que haya esa representatividad. De ese modo, toma en cuenta la posibilidad de conflicto como parte de la interculturalidad. Por tanto, apela a la definición clara de posiciones que llevarán a cabo procesos de negociación.

\section{Análisis de casos}

En el caso particular de las PIES, el concepto de interculturalidad ha dado origen a proyectos que plantean versiones visiblemente distintas de cdi. Esto es, diferencias con respecto a las formas en que habrá de llevarse a cabo el reconocimiento de las etnicidades Indígenas como productoras de conocimiento. Tal es el caso de los ejemplos aquí analizados: Brasil y México, que desde una mirada inicial no podrían parecer más opuestos.

En el caso de Brasil, las PIES se resumen en el Programa de Apoio à Formação Superior e Licenciaturas Indígenas, conocido como PROLIND. 
Este programa fue implementado en 2005, aunque derivó de críticas al programa Diversidade na Universidade (PDU) creado tres años antes con financiamiento del Banco Interamericano de Desarrollo (BID). Inicialmente, el PDU contempló de manera muy modesta acciones dirigidas a la población Indígena, siendo más una respuesta para el movimiento afrodescendiente. Dichas acciones consistieron en el financiamiento de proyectos innovadores de cursos (PICs) los cuales se refieren a la preparación de jóvenes de poblaciones en desventaja socioeconómica para la presentación del examen de ingreso a la universidad.

El ministerio de educación (MEC), a través de la CoordenaçãoGeral de Apoio às Escolas Indígenas (CGAEI), negoció entonces con el BID la creación de una rama indígena dentro del PDU que terminó siendo el PIC-Professores Indígenas (PIC-PI) donde dos proyectos piloto fueron financiados, uno en el estado de Bahía y otro en el estado de Mato Grosso. Estos proyectos inovaron los PICs en dos aspectos: incluyeron la noción de formación semi-presencial con el fin de combatir el problema de la distancia entre las aldeas y los centros de formación, y crearon becas comunitarias.

El PROLIND es descrito como un esfuerzo compartido entre el MEC, los movimientos indígenas y algunas universidades. Fue creado como una respuesta a las dificultades de compatibilidad entre PDU y las necesidades de los Pueblos Indígenas. Así, a través de procesos de consulta con organizaciones indígenas con respecto a posibles mejoras del PDU, se incorporó la noción de conocimientos indios a la cuestión de acceso a la universidad, reconociendo que las particularidades culturales son de primera importancia para las luchas de autonomía relacionadas con las Tierras Indígenas. Este programa opera a través de convocatorias (Editais) para que universidades públicas implementen proyectos de cursos de formación de maestros indígenas. Dichos cursos adquirieron el nombre de Licenciaturas Indígenas. Entre 2005 y 2009, 34 proyectos en 26 instituciones de educación superior fueron beneficiados con el financiamiento PROLIND.

Por su parte, en México las PIES no han estado conectadas tan directamente con los movimientos sociales ni exclusivamente limitadas 
al campo de formación de maestros. La interculturalidad se ha materializado en este caso como una política de diversificación del sistema de educación superior. Puntualmente, ha consistido en la creación de un nuevo tipo de instituciones educativas, conocidas como universidades interculturales. Creado oficialmente en 2003, este subsistema buscaba responder a las sugerencias del Plan Nacional de Educación 2001-2006 del gobierno Fox en el que se propuso la diversificación como medida para promover equidad y calidad.

Los criterios para definir dónde se localizarian esas universidades tienen que ver con la identificación de regiones con alta presencia de población indígena y una oferta pobre o nula de formación terciaria. El elemento que parece diferenciar al modelo intercultural de otras universidades es el área concebida como 'vinculación con la comunidad', un eje que busca conectar la docencia y la investigación con las necesidades y problemas de las comunidades donde la universidad está asentada. Entre 2003 y 2006, ocho universidades interculturales fueron creadas en diferentes estados del país.

Ahora bien, los dos casos de análisis pueden caracterizarse a grosso modo a través de dos ejes: 1) la definición e identificación de los beneficiarios de las politicas; y 2) el conocimiento de la interculturalidad que es producido dentro del marco de las mismas. La reflexión de este artículo presta mayor atención al segundo en tanto se ubica como un aspecto que da cuenta de la acción intelectual de los sujetos indígenas. Cabe aclarar que ambos deben ponerse en relación.

\section{Definición e identificación de beneficiarios de las PIES}

A pesar de que en ambos casos las políticas de interculturalidad surgen como solución contra la exclusión de poblaciones indígenas en educación terciaria y forman parte de una ola de democratización en torno a las diferencias étnicas, solo los programas creados por PROLIND en Brasil mantienen un foco exclusivo sobre Pueblos Indígenas. En ese caso, para formar parte de una licenciatura intercultural se debe ser indígena; esto porque tales proyectos se relacionan directamente con 
el objetivo de las luchas indígenas en Brasil sobre autonomía cultural y territorial, donde contar con maestros provenientes de las propias comunidades resulta prioritario.

Las universidades interculturales en México han sido creadas mayormente en regiones rurales que muestran alta presencia indígena; sin embargo, no contemplan un ingreso exclusivo y están abiertas a toda la ciudadanía. Así, la centralidad de lo indígena se expresa dentro de los contenidos y objetivos de la opciones de formación ofrecidas por tales instituciones; se trata de formar profesionales que conozcan y valoren los conocimientos y prácticas de las comunidades indígenas y que sean capaces de identificar sus aportaciones a la sociedad en general y generar proyectos para el mejoramiento de las condiciones de vida de las mismas. Para ello, se enfatiza el papel del conocimiento brindado por las ciencias occidentales como un complemento necesario.

Lo que este eje nos permite ver es que en estos casos tenemos distintas versiones de interculturalidad que parecen oponerse drásticamente. Por un lado, se tiene una versión en la que los sujetos target de la política son exclusivamente indígenas, de modo que la interculturalidad puede ser claramente ubicada como parte de proyectos de una etnicidad específica. Por otro lado, está la versión en la que la interculturalidad es vista como una opción para todos. La primera versión parecería contradictoria en el sentido en que se limita a grupos específicos y por ende, no pone a dialogar a las diferentes perspectivas de conocimiento, mientras que la segunda versión parecería coherente puesto que su ejecución implica el encuentro de diversos grupos étnicos. La cuestión sin embargo, es mucho más compleja que ese razonamiento.

Ahora bien, dado que la formación de licenciatura intercultural en Brasil es exclusiva de Pueblos Indígenas, los modos para identificar a los beneficiarios giran en torno al establecimiento de límites claros. Aquí interviene la acreditación pública de la membresía grupal, pero esta no se lleva a cabo como un proceso de auto-adscripción individual de orden liberal. Mientras que para las políticas de acción afirmativa (reserva de lugares) en universidades públicas, los candidatos 
deben acreditar ante la universidad correspondiente su membresía por medio de cartas de autoridades comunitarias y/o certificaciones de la Fundación Nacional del Indio (FUNAI), en el caso de las licenciaturas interculturales, el proceso tiene otras rutas debido a que el sujeto beneficiario es de orden colectivo.

En este caso la identificación de los beneficiarios concierne a un proceso de larga duración que abarca la historia de las relaciones entre Pueblos Indígenas y sociedad no indígena. La identificación se produce durante el proceso de evaluación de los proyectos para su financiamiento. La etnicidad estaría demostrada entonces a través de un vínculo histórico: relaciones de trabajo sociopolítico en las comunidades con otros actores como académicos y departamentos de gobierno. Sin embargo, hay que aclarar que si bien esta identificación comienza con el diseño del proyecto, este no necesariamente ha surgido a partir de las convocatorias, sino por otros lados y de manera previa. Como política, la interculturalidad puede entonces conectar diversas experiencias y en esa labor de conexión se identifica a los beneficiarios.

El PROLIND financia proyectos educativos cuya condición básica es que deben ser diseñados en una colaboración universidad-comunidad, por tanto reconoce el trabajo político de las poblaciones indígenas como algo valioso y útil y no solo acredita la pertenencia étnica de individuos. Así, es el mismo concepto de licenciatura intercultural, una aportación nacida de la iniciativa del trabajo conjunto de organizaciones con comunidades indígenas, lo que garantiza la pertenencia étnica de los beneficiarios. En este sentido, la interculturalidad como política de educación superior hace operable la noción de Pueblos.

En el caso de México, los procesos de identificación de los beneficiarios se visibilizan en la planificación de las instituciones interculturales. Es en dicha planificación, específicamente al decidir la ubicación de una universidad intercultural, donde el sujeto beneficiario es identificado. Esa identificación previa puede operar precisamente porque el funcionamiento de estas universidades está basado en una política de puertas abiertas, esto es, el único requisito que se exige para ganar acceso a ellas es el certificado de estudios previos como 
acreditación de formación suficiente para la educación superior. No se han establecido criterios académicos, ni criterios culturales. En ese sentido, la identificación ha sido tarea de los creadores de la política pública. Esta identificación alcanzada dentro del proceso de planificación, al enfatizar criterios geográficos desde los que se ubican regiones carentes de oferta de educación superior, localiza al sujeto beneficiario más en términos de clase social que de etnicidad. Lo indígena aparece en gran medida debido a la asociación común de lo rural-indígena con la desventaja socioeconómica.

\section{El conocimiento de la interculturalidad producido en cada caso}

\section{Nota metodológica:}

Como se ha mencionado, las PIES enfatizan la participación activa de los sujetos indígenas en su formación, esta participación no se refiere solo a los asuntos dentro del aula, sino también a la intervención en campo y a la reflexión acerca de su propia formación enmarcada como intercultural. De ese modo, la publicación de materiales académicos es también un asunto en el que los sujetos en formación participan; trascienden así la posición de demandar reconocimiento para ocupar una posición de sujetos reconocidos que demuestra su capacidad de actuar y por ende, de merecer el reconocimiento.

Parte importante de estos materiales se concentra en las acciones de los propios sujetos como 'estudiantes interculturales', de modo que también dan cuenta de la perspectiva con la que desde las PIES se concibe al conocimiento de la interculturalidad en su carácter de solución social contra la injusticia. Específicamente, se analizaron documentos académicos desarrollados por estudiantes indígenas de los programas de formación superior intercultural. Los documentos fueron obtenidos de publicaciones periódicas (revistas y gacetas), libros y programas de radio; posteriormente se seleccionaron aquellos cuyo tema central era el mismo modelo de formación intercultural. Se analizaron 88 documentos para el caso mexicano y 75 documentos para el caso brasileño. 
La estrategia de análisis trata en este caso al cdi como un conjunto de textos públicos analizables a través del tipo de trabajo ideológico que realizan (Ortner, 2006). En ese sentido, la pregunta guía es: ¿qué tipo de formaciones ideológicas se construyen en y a través de dichos textos? Esto significó prestar atención a la perspectiva con que se produce el cdi (distal-representacional o proximal-performativa) y de acuerdo con ello, identificar el papel que se asigna al sujeto de conocimiento y al alcance espacio-temporal de la solución llamada educación intercultural.

\section{El conocimiento de la interculturalidad y diferenciación de casos}

Como se mencionó, el cdi no es descripción objetiva ni agrupación de demandas políticas concretas, sino un proceso reflexivo sobre las mismas PIES y sus visiones de conocimiento y de sujeto cognoscente diferenciado. En este aspecto se observan importantes contrastes entre los casos analizados.

Los resultados de esta investigación permiten caracterizar al conocimiento de la interculturalidad producido en Brasil como un conocimiento que actúa de forma proximal y de ese modo es capaz de trazar conexiones con otros proyectos que implican procesos dinámicos y estratégicos de la etnicidad. Por el contrario, en el caso mexicano el conocimiento de la interculturalidad se caracteriza por operar desde perspectivas distales de modo que la conexión con otros proyectos de formación étnica es más bien limitada. A continuación se aclaran en mayor detalle estas categorizaciones.

En gran medida, decir que el conocimiento de la interculturalidad se basa en aproximaciones distales o proximales implica identificar hasta dónde las políticas de interculturalidad, como soluciones sociales, operan con perspectivas dinámicas o estáticas de la etnicidad y el reconocimiento. En otras palabras, es ver hasta dónde estas políticas, que se enmarcan en procesos de democratización, se reconocen y se 
construyen como portadoras de reclamos políticos y líneas de acción estratégicas dentro de las estructuras de conocimiento, o bien, hasta dónde buscan distanciarse de ello.

Categorizar al conocimiento de la interculturalidad como proximal, como se sugiere para el caso de Brasil, significa identificarlo como un resultado 'parcial'. Esto es, dentro de los conocimientos que los proyectos de licenciaturas interculturales producen se puede identificar que la noción de interculturalidad juega un papel complementario, aunque muy importante, para llevar a cabo un proyecto más grande dentro de los procesos de formación étnica en Brasil. La interculturalidad como solución social es pensada no como una solución total o final para las demandas indígenas en este país, que como es sabido giran en torno al reconocimiento de derechos territoriales. Más bien, esta se ha articulado con ellas y juega el papel de colaborador.

Por ello, no es limitado que la politica de interculturalidad se concentre exclusivamente en la formación de profesores indígenas; esto nos deja ver que el conocimiento de la interculturalidad busca un ensamble con otras politicas, trayectorias y estrategias; sola no puede hacer mucho por la causa indígena. Así, si por un lado, la formación de los primeros grados de enseñanza debe ser tarea exclusiva de los propios pueblos, también es necesario que estos pueblos adquieran otros conocimientos -ubicados como técnicos- que servirán para producir interacciones con el no indio y no quedar aislados ${ }^{4}$.

Pero la parcialidad de la interculturalidad es sistemáticamente visible en otros aspectos. La producción de conocimiento desde esta política muestra características que permiten reconocer a la interculturalidad como una conexión parcial, es decir, como una política del llegar-a-ser. Al tratarse de un proyecto de formación de formadores, el tiempo de la interculturalidad es siempre futuro, siempre abierto a las posibilidades. En ese sentido, el significado de poner en relación diferentes conocimientos y diferentes grupos étnicos rebasa la dimensión temporal en la que se ejecutan los proyectos de licenciatura intercultural, no se queda en

\footnotetext{
4 Esto a través del recurso de políticas de acción afirmativa en universidades y en la misma preparación de profesores indigenas en disciplinas técnicas.
} 
el presente. Pero también rebasa la dimensión espacial. Si bien el encuentro entre indígenas y no indígenas durante la formación ocurre en el contexto educativo, tanto en universidades como en aldeas, el objetivo buscado es la aplicación de ese encuentro a gran escala de modo que los Pueblos Indígenas dialoguen con la sociedad no indígena sin tener desventajas producidas por asimetrías de conocimientos.

Esto conduce a dos características más; primeramente, que el sujeto colectivo indígena que se busca constituir es uno que habla(rá) frente a una sociedad-Estado en términos concretos de demanda política. Parte importante del conocimiento que se produce construye sujetos que habrán de demandar la adquisición y cumplimiento de derechos como Pueblos. Para ello, el cdi enfatiza la importancia de conocer la serie de documentos legales que respaldan una educación diferenciada para Pueblos Indígenas; y además divulga formas de acción por parte de los sujetos donde se deja claro que los logros requieren organización política. Este énfasis en un sujeto político preparándose para demandar derechos nos conduce al segundo aspecto: a la opción intercultural no se le borra la dimensión de conflicto, más bien se deja claro que la relaciones interculturales, aunque pacíficas, se deben perfilar dentro de un marco de etnicidad en relación de competición.

Finalmente, estas características hacen patente que, las relaciones interculturales son algo que ha de ser realizado por los sujetos, algo que depende de los intereses e interacciones de orden politico, en lugar de una solución derivada de preceptos meramente teóricos o mucho menos, de la mera existencia de diversidad cultural. Al mismo tiempo, la interculturalidad con su cercania a lo Indígena se convierte en un elemento que forma parte de la red de conexiones que constituyen a la etnicidad indigena en Brasil como una con claros límites respecto a las poblaciones no indígenas.

En el caso del cdi en México, la noción de interculturalidad se da a conocer como una solución total, capaz de ofrecer los arreglos necesarios para que el reconocimiento de las diferencias tome lugar. No se debe olvidar sin embargo, que la noción de interculturalidad en este caso adopta la idea de estar dirigida a toda la población; por tanto, 
los proyectos que puede apoyar se encuentran menos diferenciados. La opción intercultural en México revela así un marco renovado para interpretar a la nación entera; no se trata de construir sujetos diferenciados; por el contrario, la formación étnica que se promueve es la de la convivencia inmediata que suspenda las fronteras étnicas sin borrarlas definitivamente. Mientras que en Brasil es la visible división de grupos la figura central que evoca a la interculturalidad, en México lo es el encuentro de grupos.

Como en el caso brasileño, es a través de tres puntos que se propone la categorización de la opción intercultural en México como una perspectiva de totalidad y cierre: alcance espacio-temporal de la solución social, la posición de estudiante intercultural y la noción de conflicto en relación con lo intercultural. Elementos como las opciones de formación que ofrecen las universidades interculturales ${ }^{5} \mathrm{o}$ sus politicas de puertas abiertas al estudiantado, permiten ver que la noción de interculturalidad es algo que toma lugar en el momento mismo de llevarse a cabo los procesos de formación. Por supuesto que constantemente se mencionan expectativas a largo plazo como metas de esta política, sin embargo, es en el presente donde se considera que la interculturalidad se expresa. Ya que aquí la idea central es la de encuentro de grupos culturales para producir así la valoración mutua de sus prácticas de conocimiento, el escenario educativo y la comunidad, así como la comunidad como escenario educativo, brindan las posibilidades para lograr esa meta.

En ese sentido no es solo en un marco espacial delimitado que la educación intercultural se desarrolla sino también en un tiempo presente. La interculturalidad en México ya 'es' y el hecho de que se le sitúe en la acción que se lleva a cabo dentro de las universidades interculturales también significa considerar que en ese momento el reconocimiento está siendo completado, está alcanzando su cierre. Por supuesto, hay excepciones. En algunos documentos analizados, se reconoce la percepción de que la interculturalidad dentro de las mismas universidades interculturales no existe, precisamente porque los estudiantes indígenas sienten

\footnotetext{
5 Estas están basadas en la noción de pertinencia cultural. Por ejemplo: Turismo Alternativo o Lengua y Cultura.
} 
aún menosprecio por parte de, y desventajas en comparación con estudiantes no indígenas y algunos profesores.

Ahora bien, el sujeto que se construye desde esta política corresponde a un sujeto descubridor, aquel que aprende la cultura del otro y también reconoce la propia, para que de ese modo cree una opción híbrida no asimilacionista. Este sujeto se hace posible gracias a la centralidad que en este contexto se otorga a la cultura como una serie de rasgos o características que pueden organizarse a través de contenidos de aprendizaje. Además, dado que se presenta una narrativa de recuperación y rescate de las culturas indígenas en combinación con la noción de resistencia, la forma particular como se ponen en marcha las relaciones entre grupos culturales es a través de una noción de traducción. Los sujetos descubridores-rescatadores son traductores de una u otra cultura, pero también de las condiciones de convivencia intercultural en México que, debido a sus condiciones históricas, presenta pocos límites precisos (como el conocimiento de una lengua indigena) entre poblaciones indígenas y no indígenas.

Si lo central en la producción de la interculturalidad inmediata es la materia prima llamada cultura, mayormente entendida como algo que ha resistido o ha sido casi exterminado y si se tiene un contexto histórico donde la herencia indígena forma parte importante en la construcción nacional, entonces la interculturalidad como solución social tenderá a acercarse más a la idea de una solución espontánea que a la de ser producida por acción estratégica de los sujetos involucrados. El objetivo radica en formar sujetos capaces de identificar las expresiones de interculturalidad ocurridas espontáneamente y traducirlas a un lenguaje científico que les preste legitimidad. Se trata de ciencificar las prácticas comunitarias rurales-indígenas y de socializar los conocimientos científicos. Por ello, los sujetos traductores pueden ser indígenas o no indígenas: la interculturalidad existe independiente de los intereses que como grupos diferenciados pudieran presentar.

Lo anterior puede hacer suponer una visión sobre los intereses indígenas como intereses enmarcados dentro de la nación entera, la idea 
armoniosa de que la coexistencia pacífica haria que esos intereses, aunque relacionados a ciertas poblaciones, sean también beneficiosos para la nación entera. De ahí que el conflicto parece resultar una antítesis de la interculturalidad.

\section{A modo de conclusión}

El análisis sugiere que más allá de las diferencias relacionadas con las características contextuales en la construcción de políticas públicas educativas, los casos se distinguen por las perspectivas epistemológicas desde las cuales toman lugar y que son las que promueven como modelo educativo. Puntualmente, la distinción es posible a partir de las diferencias en la conceptualización de la PIES como solución social a la injusticia, en donde o bien el sujeto indígena reconocido es un sujeto que interviene en la realidad y es consciente de que puede transformarla a través de su acción politizada aún si nada está asegurado (Brasil), o bien como sujeto reconocido ocupa una posición de descubridor de la realidad, donde su intervención se deriva de la certidumbre de la racionalidad científica (México). Estas diferencias en torno al sujeto coinciden con el alcance espacio-temporal de las PIES en cada caso.

Mientras que el sujeto con acción politizada se mueve en una PIES a futuro y a largo plazo, el sujeto de la acción científica se mueve en una PIES que concibe la interculturalidad como un fenómeno que toma lugar en el momento mismo de la acción educativa haciendo sentido de la coexistencia intercultural de la nación.

De forma general, se puede entonces sugerir que los casos analizados muestran hasta cierto punto que la noción estática de cdi es compartida, mientras que a través de la dinámica las diferencias se hacen visibles, siendo el caso brasileño el que con claridad exhibe dicha vertiente. En este sentido, se sugiere que las PIES, antes que insertarse en un modelo global unificador para el tratamiento de la diferencia cultural de minorías (que sería lo que se observa a través de la 
vertiente estática del análisis del cdi), o dentro de América Latina específicamente para el tratamiento de las poblaciones indígenas, descansan más bien en definiciones históricas -no genéricas- de lo Indígena.

Así, la discusión de las diferencias en la noción de la interculturalidad como solución sociopolítica puede encontrar interpretaciones interesantes para estas a partir de la identificación de los significados acerca de 'lo indígena' en cada caso nacional. Por ejemplo, la definición territorial de los Pueblos Indígenas en Brasil hace pleno sentido con la noción del sujeto indígena reconocido en la interculturalidad, preparándose para tomar posición frente al Estado. Asimismo, la definición más culturalista de lo indígena en México coincide con la noción de un sujeto enfocado a conocer, rescatar y promover contenidos culturales sin que al final la pertenencia a un grupo étnico o a otro resulte relevante. De acuerdo con ello, se pretende continuar la investigación en torno a esta relación, planteando observar cómo las PIES, como creación sociopolítica, también contribuyen a la construcción de la diversidad cultural de las naciones latinoamericanas, trascendiendo un carácter descriptivo con respecto a ella.

\section{Referencias}

Abu-Lughod, L. (1997). The interpretation of culture(s) after television. Reflections, 59, 109-134.

Apffel-Marglin, F. (1996). Introduction: Rationality and the World. En F. ApffelMarglin y S. A. Marglin (Eds.). Decolonizing knowledge. From development to dialogue (pp. 1-39). Oxford: Clarendon Press.

Castro-Gómez, S. (2007). Decolonizar la universidad. La hybris del punto cero y el diálogo de saberes. En S. Castro-Gómez y R. Grosfoguel (Eds.). El giro decolonial. Reflexiones para una diversidad epistémica más allá del capitalismo global (pp. 79-91). Bogotá: Pontificia Universidad Javeriana - Instituto Pensar, Universidad Central - IESCO, Siglo del Hombre. 
Castro-Gómez, S. y Grosfoguel, R. (2007). Prólogo. Giro decolonial, teoría crítica y pensamiento heterárquico. En S. Castro-Gómez y R. Grosfoguel (Eds.). El giro decolonial. Reflexiones para una diversidad epistémica más allá del capitalismo global (pp. 9-23). Bogotá: Pontificia Universidad Javeriana - Instituto Pensar, Universidad Central - IESCO, Siglo del Hombre Editores.

Cooper, R. y Law, J. (1995). Organization: distal and proximal view. Research in the Sociology of Organizations, 13, 237-274.

Domingues, J. M. (2009). Global modernization, 'coloniality' and a critical sociology for contemporary Latin America. Theory, Culture \& Society, 26(1), 112-133.

Escobar, A. (1999). El final del salvaje: naturaleza, cultura y politica en la antropología contemporánea. Bogotá: Instituto Colombiano de Antropología.

Fabian, J. (1983). Time and the other. How anthropology makes its object. New York: Columbia University Press.

Grimson, A. y Semán P. (2005). Presentación: la cuestión cultura. Etnografias Contemporáneas, 1, 1-12.

Haraway, D. J. (1988). Situated knowledges: The science question in feminism and the privilege of partial perspective. Feminist Studies, 14(3), 575-599.

Haraway, D. J. (1995). Simians, cyborgs, and women. The reinvention of nature. Londres: Free Association Books.

Haraway, D. J. (2008). When species meet. Minneapolis: University of Minnesota Press.

Lander, E. (2000). La colonialidad del saber: Eurocentrismo y ciencias sociales. Perspectivas latinoamericanas. Buenos Aires: CLACSO.

Latour, B. (2004). Why has critique run out of steam? From matters of fact to matters of concern. Critical Inquiry, 30, 225-248. 
López, L. E. (1994). Educación, pluralidad y tolerancia. Apreciaciones desde la educación indígena latinoamericana. Revista Unitas, 13/ 14, 35-39.

López, L. E. (2001). La cuestión de la interculturalidad y la educación latinoamericana. En UNESCO (ed.). Análisis de prospectivas de la educación en la región de América Latina y el Caribe (pp. 382-406). Santiago: UNESCO.

Maldonado-Torres, N. (2007). Sobre la colonialidad del ser: Contribuciones al desarrollo de un concepto. En S. Castro-Gómez y R. Grosfoguel (Eds.) El giro decolonial. Reflexiones para una diversidad epistémica más allá del capitalismo global (pp. 127-167). Bogotá: Ponticia Universidad Javeriana-Instituto Pensar, Universidad Central, Siglo del Hombre.

Ortner, S. B. (2006). Anthropology and Social Theory. Culture, power and the acting subject. Durham: Duke University Press.

Pickering, A. (1995). The mangle of practice: Time, agency, and science. Chicago: The University of Chicago Press.

Quijano, A. (2007). Colonialidad del poder y clasificación social. En S. CastroGómez y R. Grosfoguel (Eds.) El giro decolonial. Reflexiones para una diversidad epistémica más allá del capitalismo global (pp. 93-126). Bogotá: Ponticia Universidad Javeriana-Instituto Pensar, Universidad Central, Siglo del Hombre.

Restrepo, E. (2004a). Politicas del conocimiento y alteridad étnica. México, D. F.: Universidad de la Ciudad de México.

Restrepo, E. (2004b). Teorias contemporáneas de la etnicidad. Stuart Hall y Michel Foucault. Popayán: Editorial Universidad del Cauca.

Strathern, M. (1991). Partial connections. Savage, Maryland: Rowman \& Littlefield.

Ströbele-Gregor, J. (2010). Educación intercultural bilingüe en América Latina - ¿Una contribución a la construcción de una sociedad democrática incluyente? En J. Ströbele-Gregor, O. Kaltmeier, y C. Giebeler (Comps.) Fortalecimiento de organizaciones indigenas en América Latina. Construyendo interculturalidad: Pueblos indigenas, educación y politicas de identidad en América Latina (pp. 8-14). 
Bielefeld: Deutsche Gesellschaft für Technische Zusammenarbeit y Zentrum für Interdisziplinäre Forschung - Universität Bielefeld.

Trejo, G. (2006/2000). Etnia e mobilização social: Uma revisão teórica com aplicações à „quarta onda“ de mobilizações indígenas na América Latina. En J. M. Domingues y M. Maneiro (Orgs.). América Latina Hoje (pp. 227-275). Rio de Janeiro: Civilização Brasileira.

Turnbull, D. (1997). Reframing science and other local knowledge traditions. Futures, 29(6), 551-562.

Wallerstein, I. (1996). Social science and contemporary society. The vanishing guarantees of rationality. International Sociology, 11(7), 7-25.

Wallerstein, I. (2004). The uncertainties of knowledge. Philadelphia, PA: Temple Univ. Press.

Walsh, C. (2002). The (re)articulation of political subjectivities and colonial difference in Ecuador: Reflections on capitalism and the geopolitics of knowledge. Nepantla: Views from South, 3(1), 61-97.

\section{Cómo citar este artículo}

Martínez-Cortés, M. A. (2015). ¿Descubrir o intervenir? El conocimiento de la interculturalidad en la educación superior de Brasil y México: politicas y sujetos interculturales como objetos de reflexión y conocimiento. Universitas Humanistica, 80, 159-185. http://dx.doi.org/10.11144/Javeriana.UH80.dici 